\title{
Fatigue behavior of Nitinol medical devices under multi-axial non-proportional loads
}

\author{
Francesca Berti ${ }^{1,2}$, Pei-Jiang Wang ${ }^{2}$, Andrea Spagnoli ${ }^{3}$, Carlo Guala ${ }^{1}$, Francesco \\ Migliavacca ${ }^{1}$, Giancarlo Pennati ${ }^{1}$, Elazer R Edelman ${ }^{2}$, Lorenza Petrini ${ }^{4 *}$ \\ ${ }^{1}$ Laboratory of Biological Structure Mechanics, Department of Chemistry, Materials and Chemical \\ Engineering "Giulio Natta", Politecnico di Milano, Piazza Leonardo da Vinci 32, 20133 Milano (Italy) \\ ${ }^{2}$ Biomedical Engineering Center, Institute for Medical Engineering \& Science, Massachusetts Institute \\ of Technology, Cambridge, MA, USA \\ ${ }^{3}$ Department of Engineering and Architecture, Università di Parma, Parco Area delle Scienze, 181/A - \\ 43124 Parma (Italy) \\ ${ }^{4}$ Department of Civil and Environmental Engineering, Politecnico di Milano, Piazza Leonardo da Vinci \\ 32, 20133 Milano (Italy)
}

\begin{abstract}
Nickel-Titanium alloys (Nitinol) are widely used for biomedical applications. Peripheral stents are almost exclusively composed of Nitinol, as its superelasticity is suited for minimally-invasive insertion and durable effect. After crimping and deployment stents undergo cyclic multi-axial loads imposed by vascular and lower-limb motion (e.g. axial compression, bending, and torsion). This complex mechanical environment could lead to metal fatigue and device fracture, with possible severe consequences (e.g. in-stent restenosis). Standard regulations require experimental verification of stent fatigue behaviour for preclinical assessment, but no exact indications are provided to direct the load combination. Moreover, different fatigue criteria were developed for common metals to predict fatigue endurance, but no criteria were specifically defined for the unique thermo-mechanical properties of Nitinol. This study investigated the role of cyclic multi-axial loading conditions on different stent geometries, looking at how they affect the stress/strain distribution along the device and how different criteria may affect the fatigue prediction (e.g. the standard Von Mises alternate approach and other critical plane approaches). Then, a preliminary experimental fatigue campaign was performed in agreement with the numerical simulations in order to compare the numerical predictions with the experimental results. The result suggest that the critical plane approaches are more reliable than the standard Von Mises criterion.
\end{abstract}

\section{Introduction}

Peripheral stents are almost exclusively composed of Nitinol, as its super-elasticity is suited for minimally invasive insertion and durable effect [1]. After crimping and deployment, stents undergo cyclic multiaxial loads imposed by vascular and lower-limb motion: this complex load combination might lead to metal fatigue and device fracture, with severe clinical consequences which urge a clear indication of reliable methods for predicting their 
fatigue behavior [2]. Nitinol stents fatigue assessment is still an open issue and no univocal indications are available due to the peculiarity of the material and the complexity of the device geometry. Regulatory bodies require experimental campaign to verify the device fatigue endurance, also known as "testing for survival": a common threshold is set at $10^{8}$ cycles, which is representative of 10-years activity [3]. Since experiments are expensive and time-consuming, fatigue assessment based on the use of validated numerical models has been recognized as a key procedure for predicting safety conditions of stents, in particular when a range of in vivo-like conditions is applied. However, the results of finite element analysis (FEA) must be interpreted through fatigue criteria to assess the risk of failure, yet no unequivocal indication for Nitinol device failure remains available. Four different fatigue criteria, originally formulated for standard metals, were used in this study to evaluate the fatigue behavior of a Nitinol stent undergoing multi-axial loads and validated experimentally.

\section{Materials and methods}

\subsection{Numerical simulations}

Two FE models resembling the commercial Absolute Pro® (Abbott Vascular, Santa Clara, CA) and Complete ${ }^{\circledR}$ SE (Medtronic Vascular, Santa Rosa, CA) stents were employed (Fig. 1a). In the text, they have been addressed as stent $A$ and $C$ respectively. Details regarding the models geometry, discretization, and material properties are available in [4]. Stent crimping and implantation into the vessel introduces a state of non-zero mean stress/strain that influences the following stent deformations induced by vascular and lower-limb motion. In our numerical simulations, the stent was analyzed in its expanded configuration with a preload being applied to introduce non-zero mean stress/strain state, resembling realistic implantation scenarios. Then, a combination of axial compression, bending, and torsion loads, inspired from literature physiological data, was applied [5]. The choice of the analysis in the expanded configuration allowed to avoid all the complexities related to the contact with the artery wall that makes more difficult a reliable comparison between numerical and experimental results. In particular, an in-phase sinusoidal path oscillating between maximum and minimum values corresponding to $\pm 2 \%$ axial strain, $\pm 10 \mathrm{~mm}$ of curvature radius, and $\pm 3 \% \mathrm{~cm}$ of torsion angle was applied first (P1). Then, a different load scenario (P2), with $\pm 2.3 \%$ axial strain, $\pm 13 \mathrm{~mm}$ of curvature radius, and $\pm 4 \% \mathrm{~cm}$ of torsion angle was considered. The results, in terms of stress/strain tensors extracted from the centroids of the elements at each increment of the fatigue cycle, were used to predict the fatigue behavior of the stent. Four different fatigue criteria, based on different mechanical assumptions, were considered to compute a fatigue index (FI) based on the loads acting on the stent. The standard von Mises (VM) criterion was compared to three critical plane approaches. The VM index is the equivalent alternate strain, calculated from the principal alternate strain $\Delta \varepsilon_{1}, \Delta \varepsilon_{2}, \Delta \varepsilon_{3}$ (Eq.1). The Fatemi-Socie (FS)[6] is a strain based criterion for shear failure mode that utilizes the maximum shear strain amplitude $\left(\Delta \gamma_{\max }\right)$ and the maximum normal stress $\left(\sigma_{n, \max }\right)$ on the plane of the maximum shear strain amplitude (Eq. 2). The Brown-Miller (BM)[7] is also a strain based criterion which combines the maximum shear strain amplitude and the maximum normal strain amplitude $\left(\Delta \varepsilon_{n}\right)$ on the plane of maximum shear strain amplitude (Eq.3). The 
Smith-Watson-Topper (SWT)[8], [9] is an energy based approach and the FI is given by the product of the maximum normal stress and the maximum normal strain amplitude, both on the plane of maximum normal strain (Eq.4).

$$
\begin{gathered}
V M=\frac{1}{2(1+v) \sqrt{2}} \sqrt{\left(\Delta \varepsilon_{1}-\Delta \varepsilon_{2}\right)^{2}+\left(\Delta \varepsilon_{2}-\Delta \varepsilon_{3}\right)^{2}+\left(\Delta \varepsilon_{3}-\Delta \varepsilon_{1}\right)^{2}} \\
F S=\frac{\Delta \gamma_{\max }}{2}\left(1+k \frac{\sigma_{n, \max }}{\sigma_{y}}\right) \\
B M=\frac{\Delta \gamma_{\max }}{2}+S \frac{\Delta \varepsilon_{n}}{2} \\
S W T=\sigma_{n, \max } \frac{\Delta \varepsilon_{n}}{2}
\end{gathered}
$$

The empirical constants $\mathrm{k}$ and $\mathrm{S}$ involved in the FS and BM criteria were taken as equal to unity in the following, as suggested by literature [10], [11].

For each numerical simulation, a set of constant life diagrams was built according to each criterion: all the FIs were calculated for each finite element of the model and their values plotted against the first principal mean strain obtaining different point clouds.

\subsection{Experimental tests}

Nitinol tubes from the same batch are laser cut into several samples having the same geometry of stents A and C (Fig.1b) and ad-hoc dog-bone specimens (Fig.1c) for mechanical and fatigue characterization of the material. The deduced material properties were used to feed the stent numerical models, the fatigue data were used to deduce the material fatigue strength at $10^{6}$ cycles [4]. The choice of this threshold is justified by the fact that Nitinol has an almost constant fatigue behavior after $10^{5}$ cycles [2]. The stent samples were used to experimentally repeat the numerical multi-axial cyclic tests. The tests were conducted at 1 $\mathrm{Hz}$ to mimic the frequency of gait. The experimental run-out was set at $10^{6}$ cycles. A customized set-up was adapted on the dynamic testing system Instron ElectroPuls E1000 (Norwood, MA): the machine axial command was transduced into multi-axial loads (axial compression, bending, and torsion) acting on the stent (Fig.1 d) in a temperature controlled environment. 
a) FE Stent $C$ model

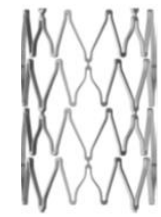

Peak-To-Peak

b)

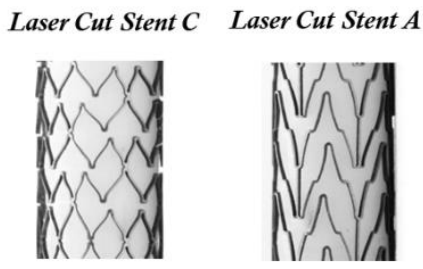

c)

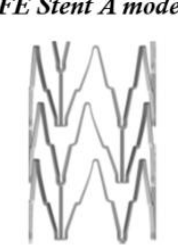

Peak-To-Valley

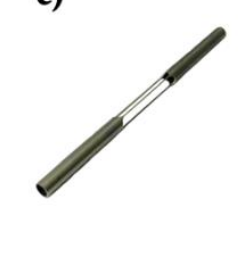

d)

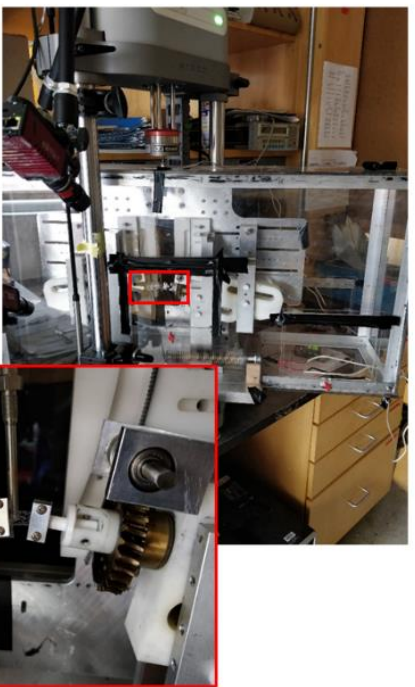

Fig. 1 a) Stent FE models; b) laser cut stent samples; c) ad-hoc developed dog-bone specimen d) experimental set-up for multi-axial loads

\section{Results}

The material fatigue strength obtained from experimental results was introduced in the constant life diagram of each criterion (Fig. 2a and $3 \mathrm{a}$ ) and used to calculate a fatigue risk factor (RF), defined as the normalized distance between the point cloud and the fatigue strength (Table 1 and 2).

The results of experimental tests on the stents were used to conform fracture induction and to determine which criteria most correlated fracture with risk prediction (Fig. $2 b$ and $3 b$ ). 

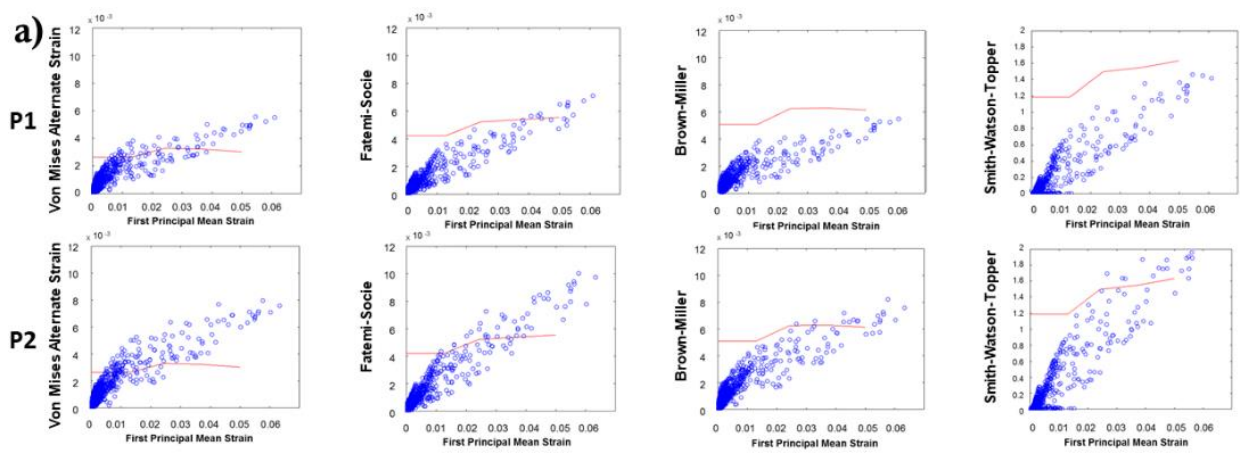

b)

\begin{tabular}{cccccc}
\hline \multicolumn{5}{c}{ FEA-Stent A } & EXP \\
\hline Case & VM & FS $(K=1)$ & $B M(S=1)$ & SWT & \\
P1 & Fail & Fail & Safe & Safe & S-S-S \\
P2 & Fail & Fail & Fail & Fail & F-F-F \\
\hline
\end{tabular}
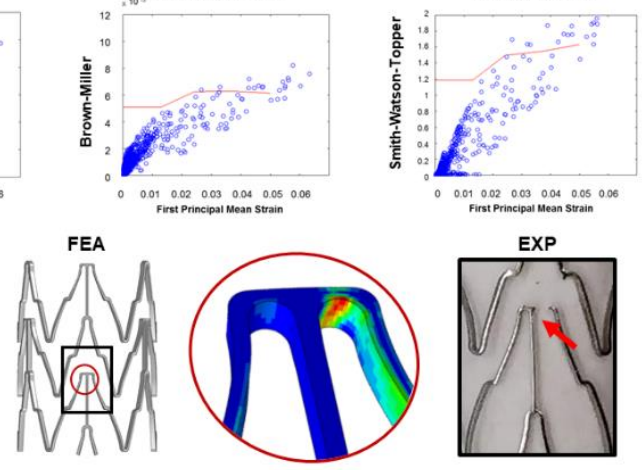

Fig. 2 Cases P1 and P2 for stent A: a) comparison of the constant life diagrams obtained computationally for each criterion and the corresponding material fatigue limit at $10^{6}$ cycles deduced from experimental results; b) numerical prediction deduced from the picture above according to the four criteria and the corresponding experimental outcome. The same fracture location was predicted and experimentally verified.
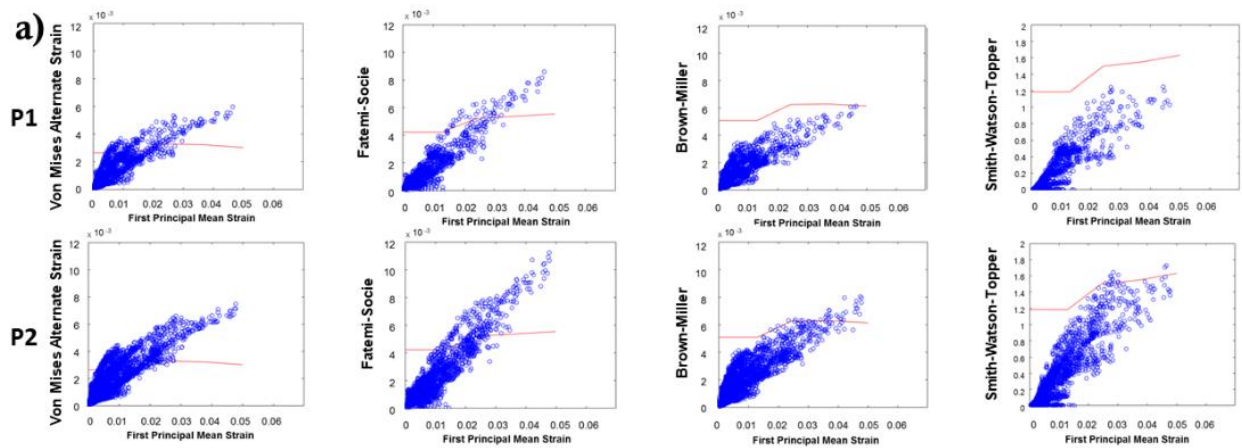

b)

\begin{tabular}{cccccc}
\hline \multicolumn{5}{c}{ FEA-Stent C } & EXP \\
\hline Case & VM & FS (K=1) & BM $(S=1)$ & SWT & \\
P1 & Fail & Fail & Safe & Safe & S-S-S \\
P2 & Fail & Fail & Fail & Fail & F-F-F \\
\hline
\end{tabular}
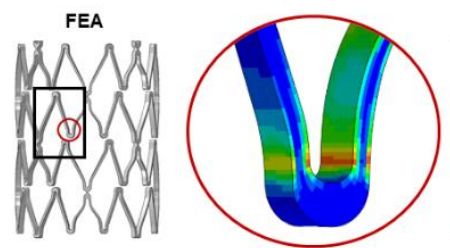

EXP

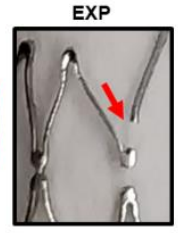

Fig. 3 Cases P1 and P2 for stent C: a) comparison of the constant life diagrams obtained computationally for each criterion and the corresponding material fatigue limit at $10^{6}$ cycles deduced from experimental results; $b$ ) numerical prediction deduced from the picture above according to the four criteria and the corresponding experimental outcome. The same fracture location was predicted and experimentally verified. 
Table 1 Risk factors for stent A and C under P1 and P2 load combinations

\begin{tabular}{|c|c|c|c|c|}
\hline & \multicolumn{5}{|c|}{ STENT A } \\
\hline Load case & VM & FS & BM & SWT \\
\hline P1 & 1.84 & 1.28 & 0.89 & 0.90 \\
\hline P2 & 2.61 & 1.81 & 1.34 & 1.33 \\
\hline & \multicolumn{5}{|c|}{ STENT C } \\
\hline Load case & VM & FS & BM & SWT \\
\hline P1 & 1.97 & 1.54 & 0.99 & 0.83 \\
\hline P2 & 2.47 & 2.02 & 1.31 & 1.09 \\
\hline
\end{tabular}

Fatigue prediction was strongly influenced by specific criterion. However, all approaches agreed in recognizing the most critical area of the stent and all the experimental fractures occurred at the same locations predicted by numerical simulations (Fig. 2b and 3b). The VM index always expresses the highest RF, being the most conservative approach.

Stent geometry is a factor greatly affecting both the macroscopic and local responses, as proved by the points cloud morphologies: stent $\mathrm{A}$ is characterized by a more compliant structure than stent $\mathrm{C}$ [4]. In addition, the fatigue plots confirmed this finding with stent A exhibiting a fewer elements reaching high deformations $(1.3 \%$ of the whole volume overcomes $3 \%$ mean strain compared to the $2.2 \%$ for the stent C) (Fig. 2a and 3a).

The experimental results indicated a better prediction capability of the critical plane approaches, in particular the BM and SWT, even if thei $r$ fatigue indices were based on different assumptions.

\section{Discussion and conclusions}

Through computational simulations, it was possible to deeply study the local response of the stents under cyclic multi-axial loads and the interesting result was that proportional loads applied to the whole stents induce non-proportional stress and strain at the local level (Figure 4). This finding is coherent with the fact that comparing different fatigue criteria, the VM resulted less reliable than critical plane approaches. Indeed, it has been shown that the accuracy of VM is commonly limited to uniaxial loading conditions whilst no insurance is given under multi-axial and non-proportional situations [12], [13].

Moreover, it is evident that the complexity of the device geometry and the strong nonlinearity of the material do not allow to know "a-priori" the stress/strain induced during cyclic loads, but computational analyses are necessary.

The coupling of experiments and numerical models allowed better understanding of the fatigue behaviour of peripheral Nitinol stents, and highlighting that the standard approach for the Nitinol fatigue assessment, i.e. employing the VM criterion, could be improved and one solution could be the usage of critical plane approaches.

Clearly, more studies involving non-proportional loads over a broader range of conditions have to be done to properly select the most reliable fatigue criterion.

Moreover, the following aspects have to be clarified.

The FS and BM indices depend on empirical constants (K, S), whose values could influence the prediction: herein they were assumed to be 1 , because an experimental confirmation was not possible.

The material fatigue strength has been assessed through axial fatigue tests on ad-hoc multiwires samples. However, stents are mainly subjected to bending. This means that during 
fatigue characterization test, all sample cross-sections are subjected to the maximum strain, while only a small portion of the cross-section (the extremities) is exercised during realistic stent loading condition. Therefore, for the same maximum strain, the risk of fracture is higher in the former case and using the material fatigue limit deduced from uniaxial tests may be too conservative.
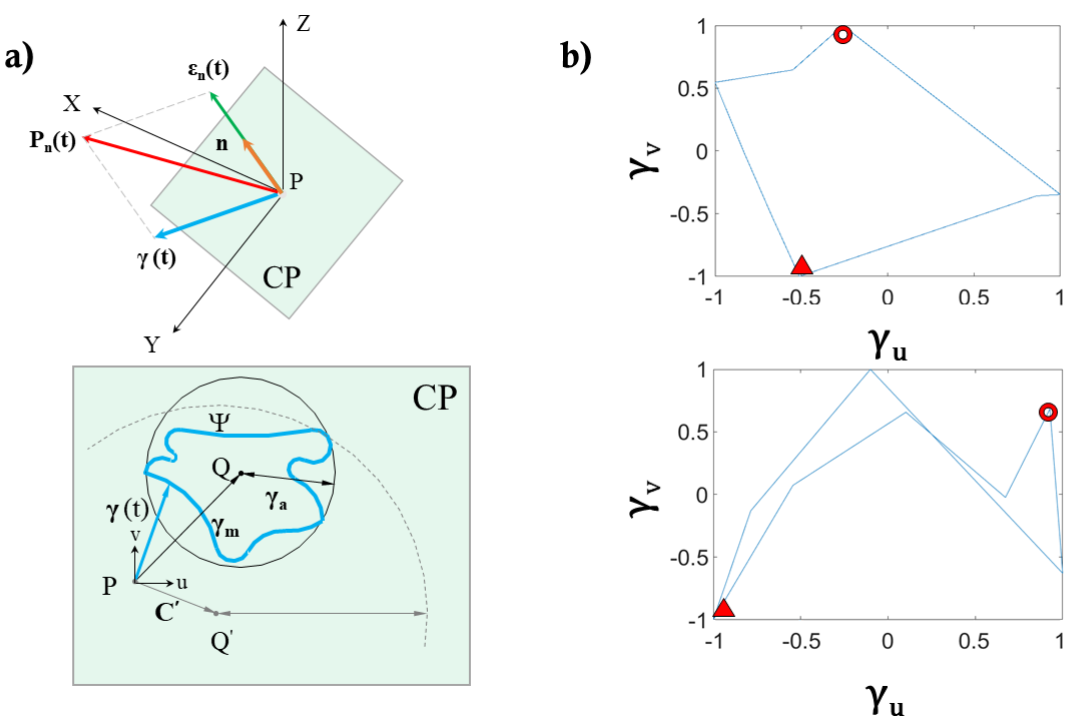

Fig. 4 a) The evolution of the shear strain vector $\gamma$ on the critical plane (CP), defined by u-v axes, during a load cycle. The point of the vector draws a non-proportional path, which is enclosed into the minimum circumference of centre $\mathrm{Q}$, whose position vector $\left(\gamma_{\mathrm{m}}\right)$ is defined as the mean shear strain while its radius $\left(\gamma_{\mathrm{a}}\right)$ represents the alternate component of the shear strain; b) normalized shear strain path for an element in the most stressed area of stent A (above) and stent C (below), calculated on the critical plane of the maximum shear strain amplitude. The triangle and the circle refer to the value at the reversals of the remote load.

\section{References}

[1] T. Duerig, a Pelton, and D. Stöckel, "An overview of nitinol medical applications," Mater. Sci. Eng. A, vol. 273-275, pp. 149-160, 1999.

[2] A. R. Pelton, V. Schroeder, M. R. Mitchell, X. Y. Gong, M. Barney, and S. W. Robertson, "Fatigue and durability of Nitinol stents," J. Mech. Behav. Biomed. Mater., vol. 1, no. 2, pp. 153-164, 2008.

[3] L. Petrini, W. Wu, E. Dordoni, A. Meoli, F. Migliavacca, and G. Pennati, "Fatigue Behavior Characterization of Nitinol for Peripheral Stents," Funct. Mater. Lett., vol. 05, no. 01, p. 1250012, 2012.

[4] D. Allegretti, F. Berti, F. Migliavacca, G. Pennati, and L. Petrini, "Fatigue Assessment of Nickel-Titanium Peripheral Stents: Comparison of Multi-Axial Fatigue Models," Shape Mem. Superelasticity, vol. 4, no. 1, pp. 186-196, 2018. 
[5] F. Ansari, L. K. Pack, S. S. Brooks, and T. M. Morrison, "Design considerations for studies of the biomechanical environment of the femoropopliteal arteries," J. Vasc. Surg., vol. 58, no. 3, pp. 804-813, 2013.

[6] A. Fatemi and D. F. Socie, "A critical plane approach to multiaxial fatigue damage including out-of-phase loading," Fatigue Fract. Eng. Mater. Struct., vol. 11, no. 3, pp. 149-165, 1988.

[7] M. W. Brown and K. J. Miller, "A Theory for Fatigue Failure under Multiaxial Stress-Strain Conditions," Proc. Inst. Mech. Eng., vol. 187, no. 1, 1973.

[8] P. Smith, K.N., Topper, T.H., Watson, "A stress-strain function for the fatigue of metals (stress-strain function for metal fatigue including mean stress effect), " $J$ Mater., vol. 5, no. January 1970, pp. 767-778, 1970.

[9] D. Socie, "Multiaxial Fatigue Damage Models," J. Eng. Mater. Technol., vol. 109, no. 4, p. 293, Oct. 1987.

[10] N. Shamsaei and A. Fatemi, "Effect of hardness on multiaxial fatigue behaviour and some simple approximations for steels," Fatigue Fract. Eng. Mater. Struct., vol. 32, no. 8, pp. 631-646, 2009.

[11] D. F. Socie, L. A. Waill, and D. F. Dittmer, "Biaxial fatigue of Inconel 718 including mean stress effects," Multiaxial Fatigue, pp. 463-481, 1985.

[12] S. Suaresh, Fatigue of Materials. Cambridge University Press, 1998.

[13] A. Fatemi and N. Shamsaei, "Multiaxial fatigue: An overview and some approximation models for life estimation," Int. J. Fatigue, vol. 33, no. 8, pp. 948958, 2011. 